\title{
Verification of the Total Zero Doppler Steering
}

\author{
Hauke Fiedler ${ }^{\# 1}$, Thomas Fritz ${ }^{\# 2}$, Ralph Kahle ${ }^{\# 3}$ \\ ${ }^{1}$ Microwaves and Radar Institute \\ ${ }^{2}$ Remote Sensing Technology Institute \\ ${ }^{3}$ German Space Operations Centre

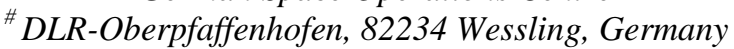 \\ ${ }^{1}$ Hauke.Fiedler@dlr.de \\ ${ }^{2}$ Thomas.Fritz@dlr.de \\ ${ }^{3}$ Ralph.Kahle@dlr.de
}

\begin{abstract}
In 2004, the Total Zero Doppler Steering was proposed to minimize the Doppler Centroid for SAR applications. It was firstly implemented in the German TerraSAR-X satellite. This satellite was launched May 2007. With the already analysed data, it is now possible to compare the predictions with the already taken data from the commissioning phase. It is shown that after an additional offset in the attitude control system of the satellite, the remaining Doppler Centroid is well below $100 \mathrm{~Hz}$ as predicted.
\end{abstract}

\section{INTRODUCTION}

The Doppler centroid is the center frequency of the azimuth spectrum recorded by synthetic aperture radar (SAR). Uncompensated variations of this Doppler centroid may cause significant errors during azimuth compression, range migration correction, and geolocalization. The calibration of SAR images will also become more difficult for high and/or varying Doppler centroids. Spaceborne SAR systems are characterized by a systematic variation of the Doppler centroid along the orbit due to the Earth's rotation. Old SAR systems designs try to minimize the Doppler centroid by a simple yaw steering method [e.g. 1, 2, 3]. In 2004, a new method was proposed which includes an additional pitch steering of the satellite, thereby reducing the Doppler centroid to theoretically Zero $\mathrm{Hz}$ [4] along the whole orbit. As an example satellite TerraSAR-X was chosen to be equipped with this steering. But, to achieve $0 \mathrm{~Hz}$ at each orbital position, the satellite has to be steered different at each position. To simplify the attitude steering, a look up table was created with 180 values of the pitch angle, yaw angle, and argument of latitude along one mean orbit. Between the values, linear interpolation is applied. The predicted Doppler centroid then ranges between $\pm 18 \mathrm{~Hz}$ at any orbital position. These values increase to $\sim \pm 100 \mathrm{~Hz}$ if the specified steering accuracy of $\pm 0.01^{\circ}$ in yaw, pitch, and roll of TerraSAR-X is applied to the calculations of the Total Zero Doppler Steering.

\section{RESULTS}

For optimal processing, radiometric accuracy and interferometric use of the products generated from TerraSAR$\mathrm{X}$ acquisitions, an exact knowledge of the beam pointing from the attitude and Doppler centroid values close to zero $\mathrm{Hz}$ are required. Deviating Doppler centroids are also a major source (besides data take start time commanding) for possible deviations in the product azimuth coverage of products and repeatability of data takes. Since the operational processing of TerraSAR-X data refers to zero Doppler times, the valid scene (and ScanSAR burst) limits in azimuth are strongly influenced by this parameter.

The TerraSAR-X Multi-Mode SAR Processor (TMSP) benefits from the high accuracy of the attitude and beam pointing knowledge by fusing this source of information ("geometric Doppler") with the Doppler centroid estimates from the raw SAR signal data. The beam pointing vectors of the selected beams (switched during Spotlight and ScanSAR acquisitions) in the antenna coordinate system are projected with the attitude information onto a precise digital elevation model to derive the Doppler centroid values for different points in the range-azimuth-time domain. The "baseband" estimates derived from the correlation of azimuth lines (Maddsen method) in several range-azimuth-time "patches" are thus not only supported by the geometric estimates in terms of selection of the right PRF ambiguity band but also stabilize the estimates in case of low backscatter areas. Also the unwrapping of the "baseband" estimates over several PRF ambiguity bands for Spotlight mode data is extremely robust due to the combination of geometric and baseband signal data.

From a slight deviation of the geometric estimates and the ones derived from the signal data, an elevation angle dependent beam squint could be detected in the very early mission phase. This problem was verified by the antenna pointing calibration teams and corrected in the attitude control system by applying offsets in the order of milli-degrees to the on-board star-tracker mounting matrices. The mean baseband (signal) Doppler centroid estimates and the ones derived from the pointing information are now aligned within the margins of the Total Zero Doppler Steering (TZDS) mode for the "operational” products. Statistical evaluation of all data takes acquired from November 15th (after the release date of the finalized “operational” TerraSAR-X ground segment systems) to December 1st verify the validity of the TZDS and the attitude steering control accuracy. Figure 1 depicts the mean Doppler centroid value of all the Stripmap datatakes taken in that time span. A remaining offset of about $30 \mathrm{~Hz}$ is visible in Figure 2. 


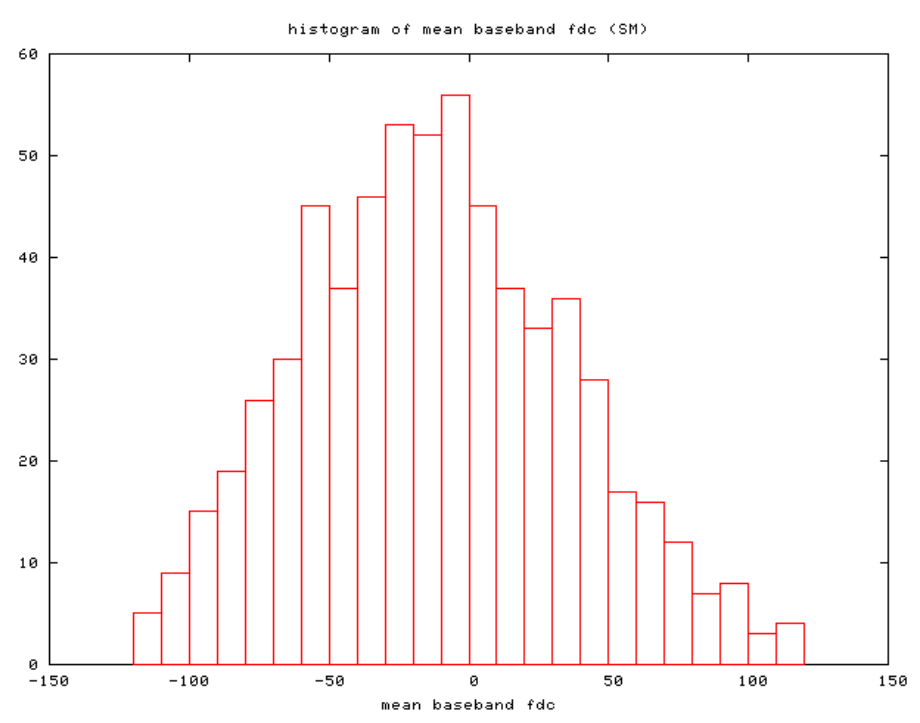

Figure 1: Mean baseband (signal) Doppler centroid of the operational data takes

The analysis of the deviations of geometric and baseband estimates also confirms the high precision in the pointing knowledge as derived from processed star tracker data and passed to the processing system as attitude products. For example, Figure 4 depicts the typical evolution of the roll, pitch and yaw Euler-deviations from the ideal attitude as described by the implemented TZDS. In general a total (3D) RMS of 0.01 deg is achieved.

However, rather strong Doppler centroid variations on short time scales are caused by the attitude control which steers the instrument well within the limits of the TZDS but leads to not perfectly smooth Doppler courses and thus slightly varying processing parameters. The variation is clearly visible in the geometric Doppler centroid estimates as well as in the baseband data as Figure 2 depicts. An optimization of the attitude control with respect to the reaction times and dead band configuration could even further optimize the pre conditions for SAR processing in the future especially in respect of Interferometric processing for the TanDEM-X mission.

The TMSP Doppler centroid estimation is accurate and works reliable. The Doppler estimates derived from the measured orbit and attitude data indicates that TS-X is well within the specified Total Zero Doppler Steering mode. The AOCS data and IOCS beam pointing information do reflect the true pointing of the beams within a few Hertz. A slightly squinted beam is not unexpected and not problematic since the initial elevation angle dependency of the beam miss-alignment has been corrected.

In Figure 2 the typical variation of the geometric Doppler estimates and the corresponding measured baseband Doppler is shown. While the mean is well within the TZDS limits, short-term variations dominate the overall Doppler course.
This correlates well with the pitch and yaw satellite attitude deviations from the ideal TZDS attitude as depicted in Figure 3.
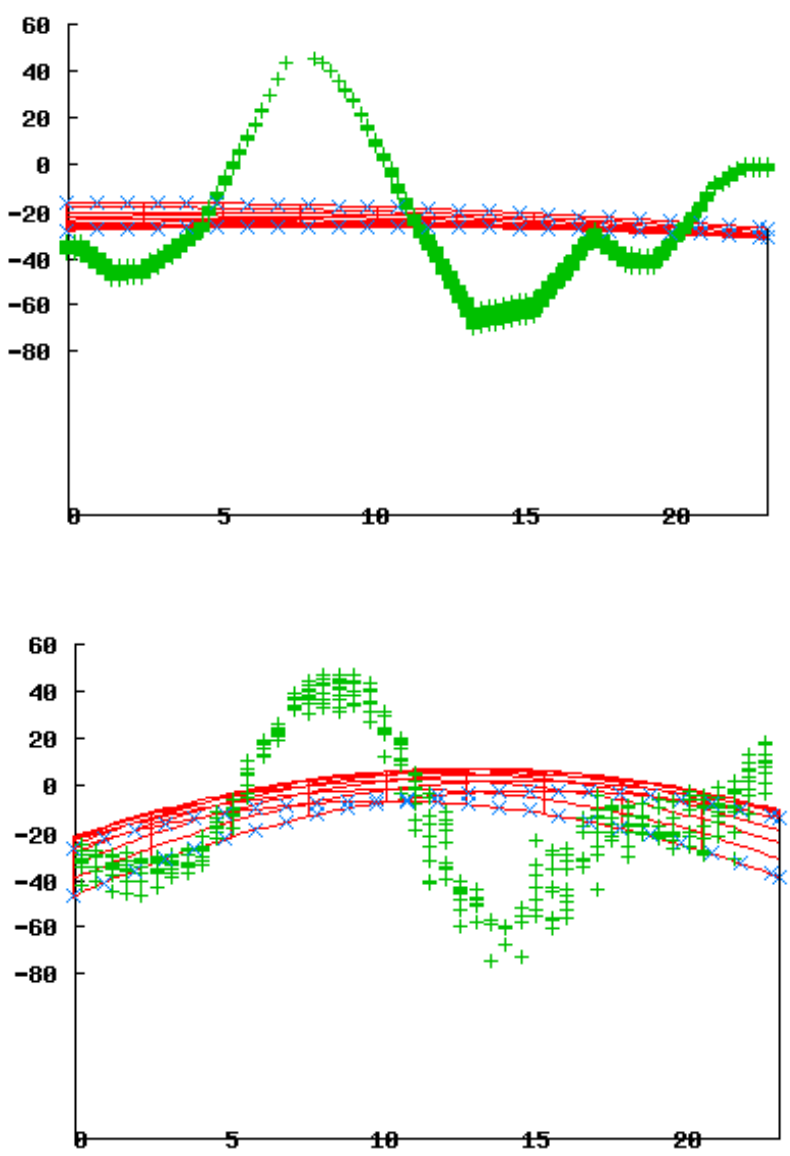

Figure 2: Typical variation of geometric (attitude-based) Doppler estimates (upper figure; $\mathrm{y}$-axis in $\mathrm{Hz}, \mathrm{x}$-axis time) and measured baseband Doppler (lower) over a 24 second long acquisition. The lines indicate polynomial fits on large time scales which are also range time dependant and thus spread out.

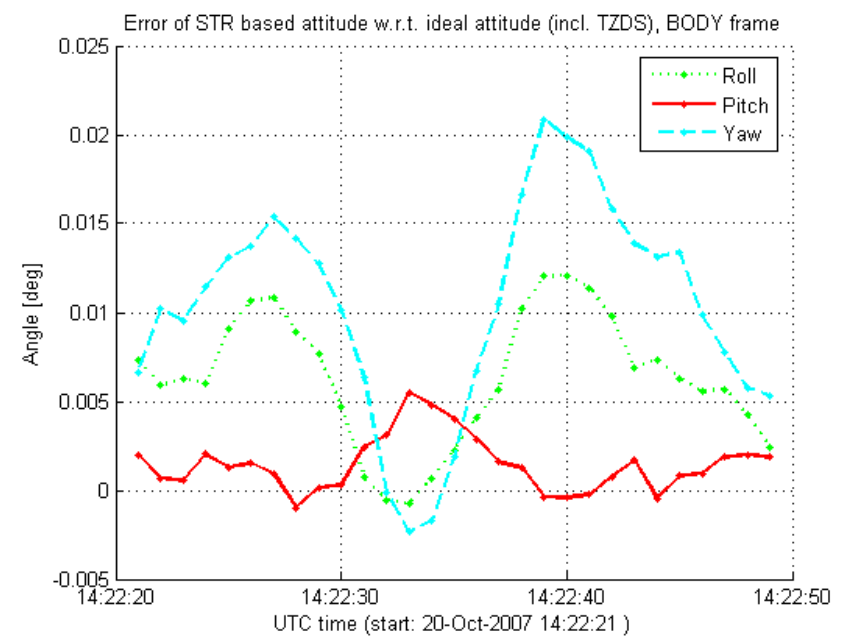

Fig. 3 Deviation of satellite (star-tracker based) attitude from reference attitude during the period of the data-take depicted in Fig. 2 (i.e. 14:22:25.914:22:48.9 UTC) 


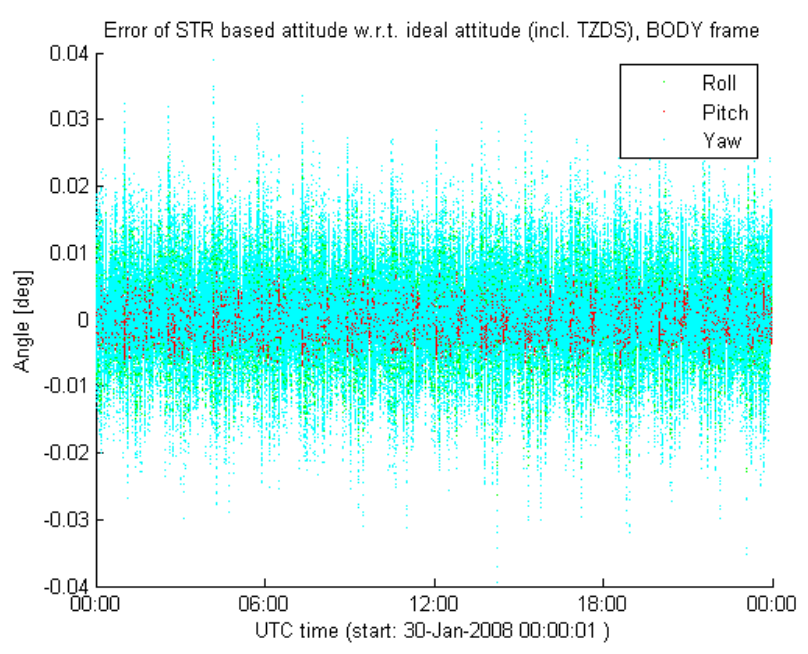

Fig. 4 Deviation of satellite (star-tracker based) attitude from reference attitude over 1-day arc. The RMS is 0.0054 deg in Roll, 0.0019 deg in Pitch, and 0.0077 deg in Yaw.

\section{ACKNOWLEDGMENT}

The authors would like to thank the TerraSAR-X team.

\section{REFERENCES}

[1] D. Just, B. Schättler, , Doppler-characteristics of the ERS-1 yaw steering mode, Proc. IGARSS , vol. 2, pp. 1349-1352, 1992

[2] H. Runge, Benefits of antenna yaw steering for SAR, Proc. IGARSS, vol. 1, pp. 257-261, 1991

[3] K. Eldhuset, Accurate attitude estimation using ERS-1 SAR raw data, Int. J. Remote Sensing, vol. 17, no. 14, pp. 2827-2844, 1996

[4] H. Fiedler, E. Boerner, J. Mittermayer, G. Krieger, Total Zero Doppler Steering - A New Method for Minimizing the Doppler Centroid, IEEE Geoscience and Remote Sensing Letters, vol. 2, no. 2, pp. 141-145, 2005 